\title{
Measured Longitudinal Beam Impedance of a Tevatron Separator
}

\author{
James L. Crisp and Brian J. Fellenz
}

\section{INTRODUCTION}

November 25, 2002

Twenty two separators are currently installed in the Tevatron. The longitudinal impedance of one of these devices was recently measured with a stretched wire. The stretched wire technique can only measure impedance below the cutoff frequency $(500 \mathrm{MHz})$. The geometry of a separator is similar to an un-terminated stripline beam position detector. The separator plates occupy a 13.5" ID vacuum tank, are 101" long, 7.8" wide, and have a 2" gap between them. The differential characteristic impedance between the plates is estimated to be $81 \Omega$ and the common mode impedance plate to ground is about $42 \Omega$.

\section{THE MEASUREMENT}

A 30 gauge (.010") tin plated copper wire was stretched through the separator and an HP 8753E network analyzer was used to measure S21, the attenuation through the device. The characteristic impedance of the wire is estimated below.

$$
\begin{aligned}
& Z_{0} \approx \frac{1}{2 \pi} \sqrt{\frac{\mu_{0}}{\varepsilon_{0}}} \ln \left(\frac{4 h}{\pi d}\right) \\
& \mathrm{Zo}=332 \Omega \quad \text { (for plate separation } \mathrm{h}=2 ”, \text { wire diameter } \mathrm{d}=.01 \text { ") }
\end{aligned}
$$

The characteristic impedance was matched to $50 \Omega$ with resistive $L$ pads at each end of the wire. The pad was made with one $330 \Omega$ resistor in series and four $220 \Omega$ resistors to ground. The driving end measured $316 / 56.3 \Omega$ and the receiving end measured 310/59.1 $\Omega$. All resistors were $1 / 4$ watt carbon. The measured attenuation through both pads agrees well with calculation at $27.65 \mathrm{db}$. The small amount of frequency structure related to the 120" length of the wire (half wavelength at $49.2 \mathrm{MHz}$ ) demonstrates the $\mathrm{L}$ pads are reasonably well matched to the characteristic impedance of the wire.

The wire itself contributes $4.344 \mathrm{db} / 100 \mathrm{ft} @ 1 \mathrm{GHz}$ from skin effect losses, Appendix 1. Wire losses are assumed to change with the square root of frequency relative to this reference point. The measurements are corrected for losses from the matching L pads and for skin effect losses along the wire.

Gravity causes the 120 " long wire to sag by about $1 / 8$ ". The separator plates were aligned vertically to avoid changes in characteristic impedance caused by this sag. One measurement was done with the wire displaced by 2 " vertically to verify the effect of the sag could be ignored.

The stretched wire technique cannot be used to measure the impedance above the cut off frequency where microwave modes can propagate independently through the device. The cutoff 
frequency is $512 \mathrm{MHz}$ for the TE11 mode in a 13.5" ID round waveguide. To verify the cutoff frequency in the separator, the coupling was measured between wire stubs placed at each end. The four $220 \Omega$ resistors to ground were left but the series $330 \Omega$ resistors were removed. The transmission (or S21) between the electric field probes placed at the ends is shown in Figure 1. The probes were 6.375 " long allowing them to extend through the bellows and 1.25 " into the tank. The coupling should increase proportionally to frequency below $463 \mathrm{MHz}$ where the probes are $1 / 4$ wavelength long.

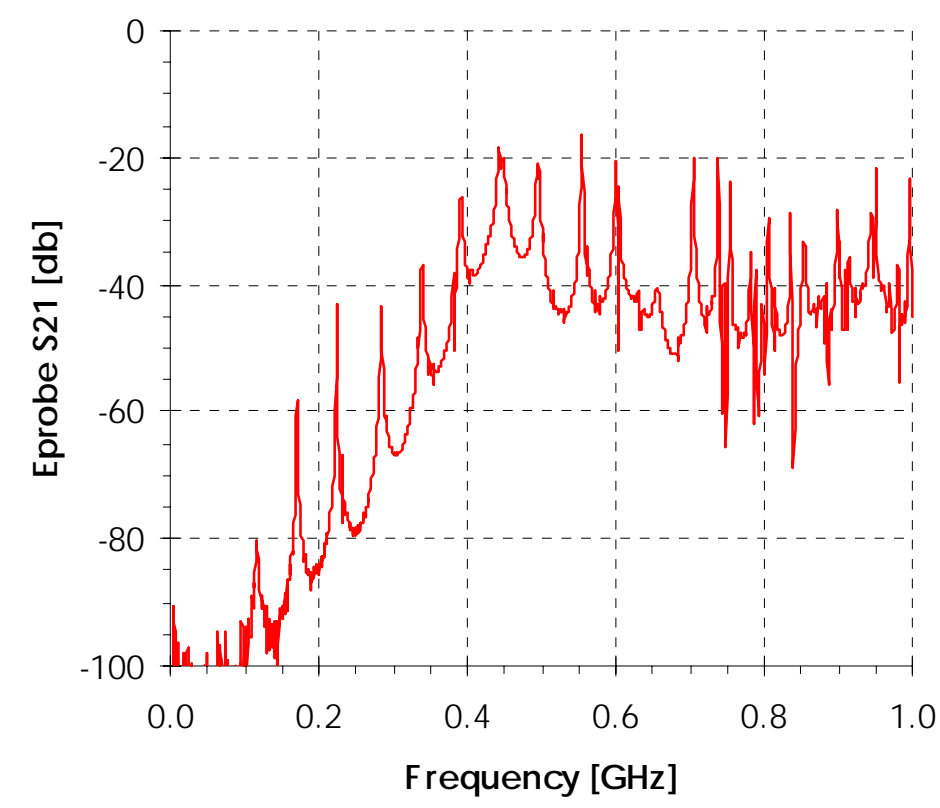

Figure 1. Coupling through the separator between electric field probes. The cutoff frequency for the vacuum tank is about $500 \mathrm{MHz}$. Coupling should increase linearly with frequency up to $463 \mathrm{MHz}$ where the probes are $1 / 4$ wavelength long.

Table 1: The first nine modes on the plate measured with the electric field probes. The frequencies correspond to the plate being multiples of $1 / 2$ wavelength long. The very first mode was not seen presumably because of the small transmission at that frequency.

\begin{tabular}{|c|c|c|}
\hline $\begin{array}{c}\text { Plate length } \mathrm{n} \\
\text { [Wavelengths] }\end{array}$ & $\begin{array}{c}\text { Resonant } \\
\text { frequency fo } \\
{[\mathrm{MHz}]}\end{array}$ & $\begin{array}{c}\text { Plate electrical } \\
\text { length nc/fo } \\
\text { [inches] }\end{array}$ \\
\hline .5 & {$[58.2]$} & {$[101.5]$} \\
\hline 1 & 116.3 & 101.5 \\
\hline 1.5 & 170.6 & 103.8 \\
\hline 2 & 223.8 & 105.5 \\
\hline 2.5 & 284.4 & 103.8 \\
\hline 3 & 338.8 & 104.5 \\
\hline 3.5 & 391.3 & 105.6 \\
\hline 4 & 445.3 & 106.0 \\
\hline 4.5 & 496.3 & 107.0 \\
\hline
\end{tabular}




\section{CALCULATING IMPEDANCE}

The lumped element model treats the separator as a simple impedance in the middle of a transmission line. S21 is a measure of the square root of the output power divided by the incident power.

$$
\begin{aligned}
& S_{21}=\left(\frac{Z_{o}}{Z+Z_{o}}\right)\left(1+\frac{\left(Z+Z_{o}\right)-Z_{o}}{\left(Z+Z_{o}\right)+Z_{o}}\right)=\frac{2 Z_{o}}{Z+2 Z_{o}} \\
& Z=2 Z_{o}\left(\frac{1}{S_{21}}-1\right)
\end{aligned}
$$
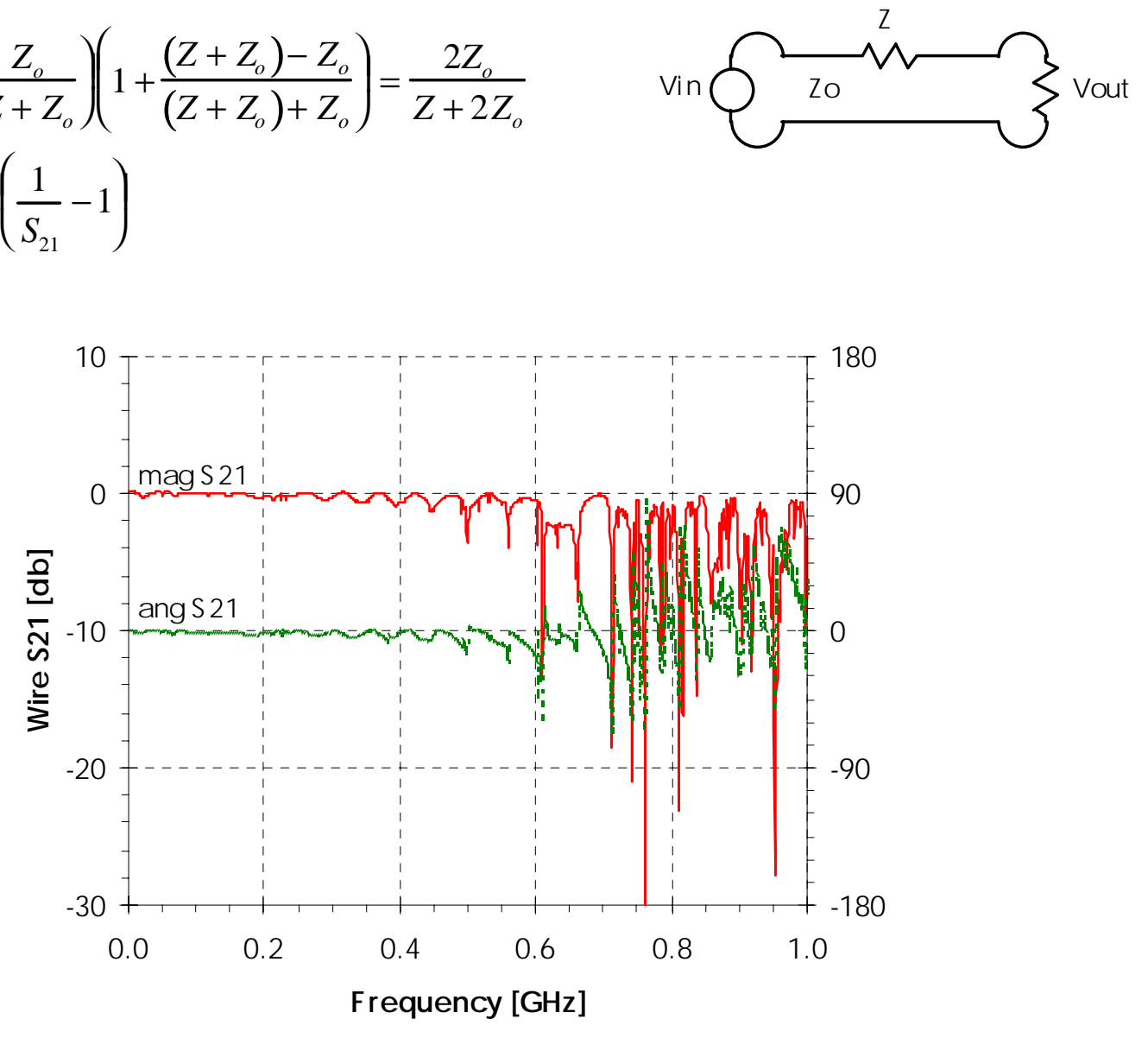

Figure 2. Transmission (S21) through the separator with the wire at the center. The attenuation was corrected for the resistive matching at the ends and for skin effect losses on the wire. The 10.22 nsec delay through the 120 " wire was removed from the phase. 


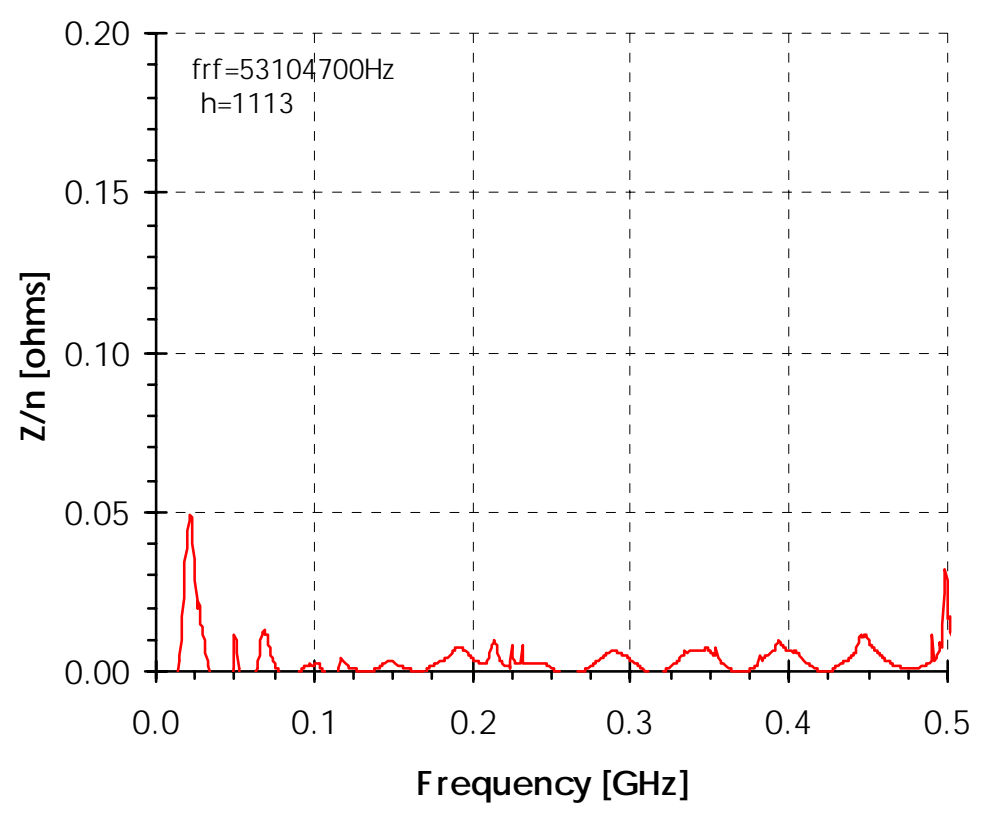

Figure 3. Longitudinal separator impedance. Virtually no longitudinal impedance is evident. The $50 \mathrm{MHz}$ periodic structure is caused by imperfect matching of the wire characteristic impedance.

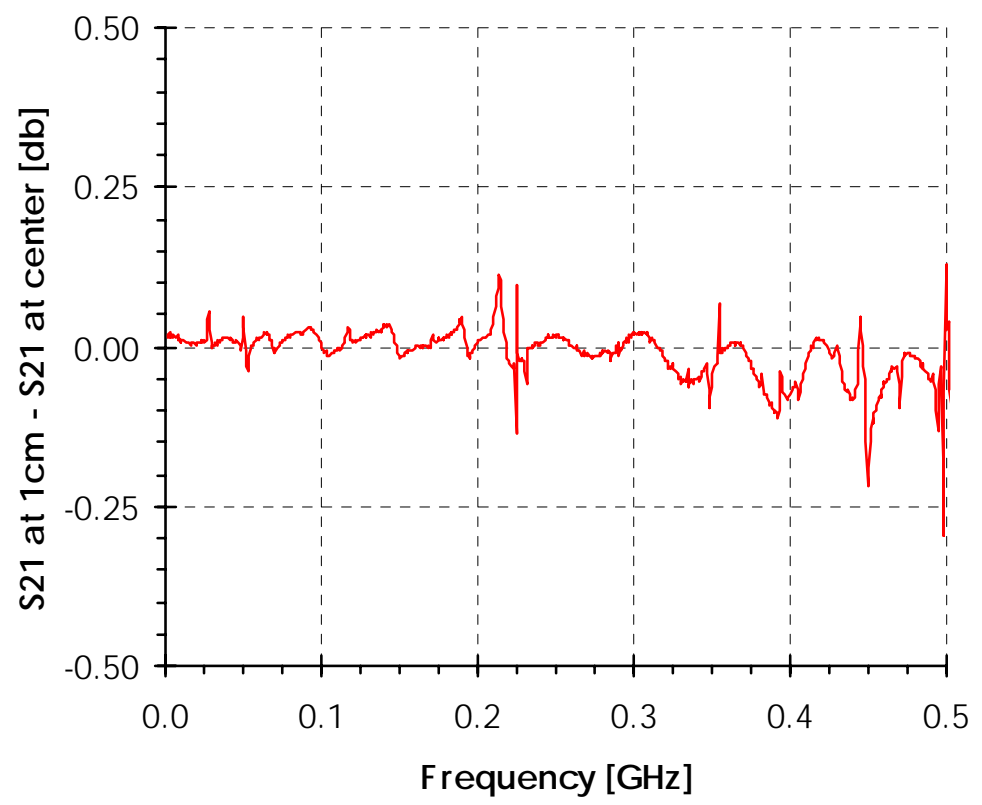

Figure 4. S21 was measured with the wire at the center and displaced $1 \mathrm{~cm}$ toward one of the plates. Some of the difference between these measurements can be attributed to the small change in characteristic impedance of the wire. The change with wire position is insignificant. 
The transverse impedance can be estimated from the longitudinal impedance, reference 2 . This approximation is intended to be used only for simple geometries. The reference specifically points out long parallel plates can have transverse impedance in the absence of longitudinal impedance. However, the longitudinal impedance measured here stems from the fields at the ends of the plates. The estimate is only included for completeness. It should not be relied upon in this case.

$$
Z_{T} \approx 2 \frac{c}{\omega b^{2}} Z_{L}
$$

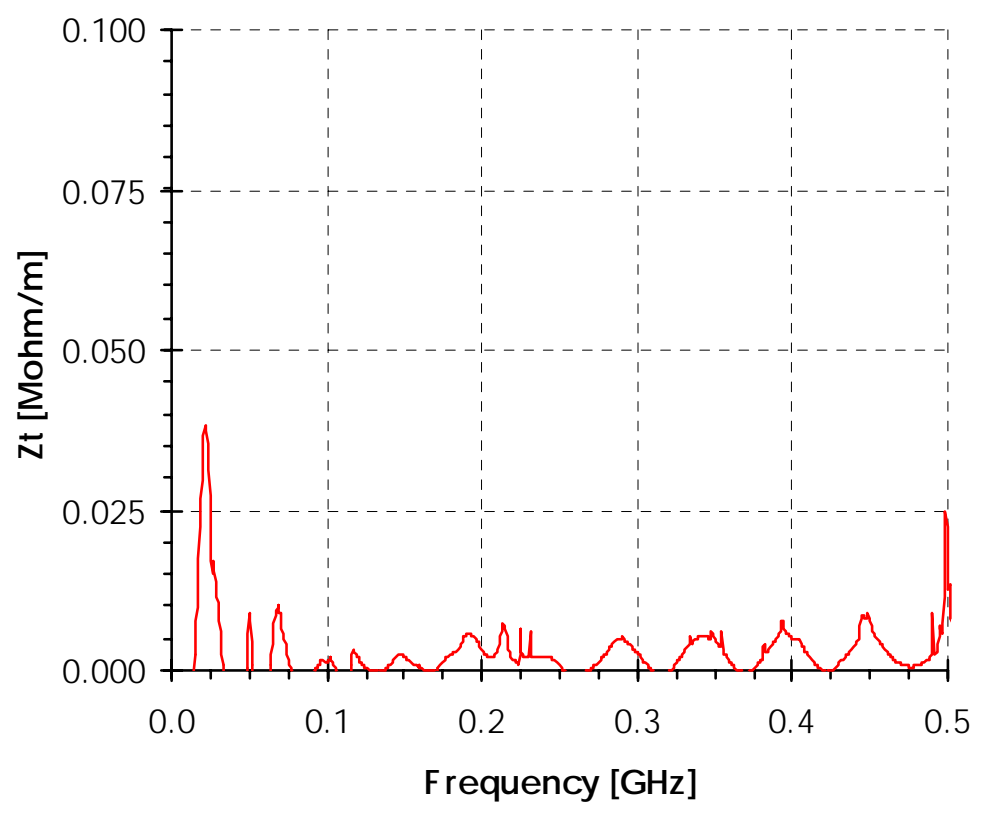

Figure 5. The transverse impedance estimated from the longitudinal impedance, Reference 2. The plot is included for completeness but should not be relied upon for reasons given in the reference.

\section{WHERE IS THE IMPEDANCE?}

\section{Longitudinal impedance}

The longitudinal electric field is nearly zero between the plates. The impedance will be dominated by the fields at the end of the plates. A stripline plate should have a family of resonant frequencies, as seen in the measurement using electric field probes. However, the effect of these modes on the beam (or stretched wire) may be negligible depending on the beam flight time and the summation of the fields at the ends of the plates. 
Stripline resonant modes:

$f_{n}=n \frac{1}{2} \frac{c}{l} \quad l=$ plate length, $c=$ propagation velocity

$n$ odd for odd modes

$n$ even for even modes

The beam flight time from one end of the plate to the other is:

$$
\Delta t=\frac{l}{c}
$$

The total accelerating voltage the beam would see for an odd mode is:

$$
\begin{aligned}
V_{\text {total }} & =V \cos \omega\left(t+\frac{l}{2 c}\right)+V \cos \omega\left(t-\frac{l}{2 c}\right) \\
& =2 V \cos \omega t \cos \omega \frac{l}{2 c} \\
& =0 \quad \text { for } \omega=2 \pi n \frac{1}{2} \frac{c}{l} \quad n \text { odd }
\end{aligned}
$$

For an even mode:

$$
\begin{aligned}
V_{\text {total }} & =V \cos \omega\left(t+\frac{l}{2 c}\right)-V \cos \omega\left(t-\frac{l}{2 c}\right) \\
& =2 V \cos \omega t \sin \omega \frac{l}{2 c} \\
& =0 \quad \text { for } \omega=2 \pi n \frac{1}{2} \frac{c}{l} \quad \text { neven }
\end{aligned}
$$

\section{DISCUSSION}

The separator geometry is virtually identical to an un-terminated stripline beam position detector. The resonant modes normally associated with stripline detectors do not contribute to the longitudinal impedance of the separator. When the beam velocity matches the propagation velocity, the sum of the electric fields at the two ends cancel. Because of this cancellation, no significant longitudinal impedance was measured in the separator.

A similar argument applies to transverse modes. Transverse fields can affect the beam along the entire length of the separator. However, the fields reverse at a resonant frequency related to the length of the plate. Because the beam velocity matches the propagation velocity, the beam sees an integer number of half periods and their effect averages to zero.

The longitudinal impedance was measured with the wire at the center and displaced $1 \mathrm{~cm}$ toward one of the plates. No significant change in impedance was seen suggesting little if any transverse impedance. 
The stretched wire technique does not provide reliable results above the microwave cutoff frequency $(500 \mathrm{MHz})$. Some structure above this frequency was seen in the wire measurements. One of the larger peaks was at $742 \mathrm{Mhz}$. The plate width is suspiciously close to $1 / 2$ wavelength at this frequency. The significance of these higher frequency components is not known.

Normally the high voltage leads extend a few meters from the separator into a series $1 \mathrm{M} \Omega$ resistor. These measurements were made with similar length high voltage cables open at one end. A $50 \Omega$ resistor is placed in series with the high voltage lead where it attaches to the plate. Two titanium sublimation pumps were also bolted to the vacuum ports. Some of the modes above $500 \mathrm{MHz}$ changed slightly with these attachments. The cables are known to be lossy at high frequency and to damp some of the higher modes on the plates. A small impedance $(\mathrm{Z} / \mathrm{n}=0.05 \Omega)$ was measured at $34 \mathrm{MHz}$. This may be associated with the plate and high voltage cables together. 


\section{REFERENCES:}

1) Sacherer, F., Nassibian, G.; "Methods for Measuring Transverse Coupling Impedances in Circular Accelerators"; Nuc. Inst. And Meth., Vol. 159, pgs 21-27, 1979.

2) Panofsky, W.K.H., Wenzel, W.A.; "Some Considerations Concerning the Transverse Deflection of Charged Particles in Radio-Frequency Fields", Rev. Sci. Instrum., Vol 27, pg. 967 , 1956.

\section{DRAWINGS:}

Electrode Support Assembly

2214-ME-261599

Overall Layout of Mounting Stands 2214-ME-276699

\section{MISCELLANEOUS DATA:}

Plate length

Plate gap

$101 "$

Plate width

$1.969 "$

ID of vacuum tank

7.8 "

Flange to flange length

Conflat vacuum flanges

$13.5^{\prime \prime}$

$120 "$

$6 "$

Differential plate impedance

$81 \Omega \quad$ estimated

$42 \Omega \quad$ estimated

Common mode plate impedance

High Voltage cable

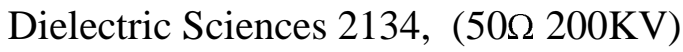

\section{STRETCHED WIRE DATA:}

Wire diameter

Wire length

Wire loss

Matching L pad

$\begin{array}{rl}.010 ” & \# 30 \text { tin plated copper wire } \\ 120 ” & \\ 4.344 & \mathrm{db} / 100 \mathrm{ft} @ 1 \mathrm{GHz} \\ 7.65 \mathrm{db} & 316 / 56.3 \Omega \text { and } 310 / 59.1 \Omega\end{array}$


APPENDIX 1: Skin effect losses in the stretched wire
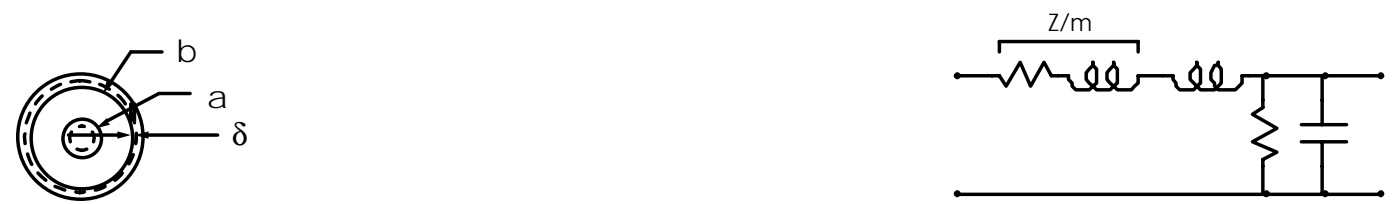

$Z_{0}=\sqrt{\frac{R+j \omega L}{G+j \omega C}} \gamma=\alpha+j \beta=\sqrt{(R+j \omega L)(G+j \omega C)}$

$R \ll \omega L$ and $G \ll \omega C$ high frequencyapproximation

$Z_{0} \approx \sqrt{\frac{L}{C}} \alpha \approx \frac{R}{2 Z_{0}}+\frac{G}{2} Z_{0} \beta \approx \omega \sqrt{L C}=\frac{\omega}{v_{p}}$

The inductive impedance equals the real impedance in a good conductor.

$Z / m \approx\left(\frac{1}{a}+\frac{1}{b}\right) \frac{\rho}{\pi \delta}(1+j)=R / m(1+j)$

skin depth in good conductor: $\delta=\sqrt{\frac{2 \rho}{\mu \omega}}$

$$
\begin{aligned}
& \text { or } \quad R / m \approx \alpha 2 Z_{0} \sqrt{f / f_{0}} \quad \text { for } \alpha \text { measured at } f_{0} \\
& \frac{\text { Vout }}{\text { Vin }}=e^{-\gamma d} \quad \text { mag }=e^{-\alpha d} \quad \text { ang }=\left(\frac{\omega}{v_{p}}-\beta\right) d
\end{aligned}
$$

A good approximation is to calculate the attenuation at $1 \mathrm{GHz}$ and assume the actual attenuation is proportional to the square root of the frequency.

$\begin{array}{ccc}\text { b 2" } & 0.0508 & \mathrm{~m} \\ \text { a .01" } & 0.000254 & \mathrm{~m} \\ \mathrm{rho} & 1.74 \mathrm{E}-08 & \Omega-\mathrm{m} \\ \mathrm{mu} & 1.26 & \mathrm{uH} / \mathrm{m} \\ \mathrm{Zo} & 317.9 & \Omega \\ \mathrm{v} & 3.00 \mathrm{E}+08 & \mathrm{~m} / \mathrm{s} \\ \mathrm{L} / \mathrm{m} & 1.07 & \mathrm{uH} / \mathrm{m} \\ \mathrm{C} / \mathrm{m} & 10.4 & \mathrm{pF} / \mathrm{m} \\ \mathrm{db} / \mathrm{neper} & 8.686 & \mathrm{db} / \mathrm{neper} \\ \text { length }[\mathrm{m}] & 2.972 & \mathrm{~m} \\ \text { attenuation } & 4.344 & \mathrm{db} / 100 \text { '@ } 1 \mathrm{GHz}\end{array}$

